\title{
Expansão das indicações geográficas para impulsionar o desenvolvimento da cachaça
}

\section{brasileira}

Expansion of geographical indications to boost the development of brazilian cachaça

Ampliación de las indicaciones geográficas para impulsar el desarrollo de la cachaza brasileña

Recebido: 16/09/2021 | Revisado: 20/09/2021 | Aceito: 21/09/2021 | Publicado: 22/09/2021

\author{
Fabiana Ilka de Queiroz \\ ORCID: https://orcid.org/0000-0002-9215-356X \\ Universidade Potiguar, Brasil \\ E-mail: fabianailka.fiq@hotmail.com \\ Tarciara Magley da Fonseca Pereira \\ ORCID: https://orcid.org/0000-0002-7570-8471 \\ Universidade Potiguar, Brasil \\ E-mail: tarciara@ufersa.edu.br \\ Juliana Bianca Maia Franco \\ ORCID: https://orcid.org/0000-0002-7566-2688 \\ Universidade Potiguar, Brasil \\ E-mail: juliana_franco_@hotmail.com \\ Cristine Hermann Nodari \\ ORCID: https://orcid.org/0000-0003-0397-337X \\ Universidade Potiguar, Brasil \\ E-mail: cristine.nodori@gmail.com
}

\section{Resumo}

O presente estudo teve como objetivo verificar a relevância da expansão das Indicações Geográficas (IG) como forma de impulsionar o desenvolvimento da cachaça brasileira. O método escolhido para esta pesquisa é de natureza qualitativa, por meio da utilização das técnicas de revisões bibliográfica e documental. A IG para a cachaça na modalidade de Indicação de Procedência para os detentores do selo é de fundamental importância no combate às falsificações e identificação para os consumidores como verdadeiras a procedência das cachaças. as vantagens do registro a valorização do saber-fazer e das técnicas tradicionais, agregação de valor, notoriedade, desenvolvimento territorial e preservação da cultura, além de demonstrar que o produto tem qualidades. Dessa forma, conclui-se que a expansão dos registros de IGs da cachaça, permitirá impulsionar o desenvolvimento da cachaça e consequentemente o desenvolvimento econômico para o país com a diminuição da informalidade na produção e comercialização da cachaça que é um produto de grande relevância no soft power brasileiro.

Palavras-chave: Indicação geográfica; Cachaça; Desenvolvimento.

\begin{abstract}
This study aimed to verify the relevance of the expansion of Geographical Indications (GI) as a way to boost the development of Brazilian cachaça. The method chosen for this research is qualitative in nature, through the use of bibliographic and document review techniques. The GI for cachaça in the form of Indication of Origin for the holders of the seal is of fundamental importance in the fight against counterfeits and identification for consumers as the true origin of the cachaças. the advantages of registering the enhancement of know-how and traditional techniques, adding value, notoriety, territorial development and preservation of culture, in addition to demonstrating that the product has qualities. Thus, it is concluded that the expansion of GI registrations of cachaça, will boost the development of cachaça and consequently the economic development for the country with the reduction of informality in the production and marketing of cachaça, which is a product of great relevance in soft Brazilian power.
\end{abstract}

Keywords: Geographical indication; Liquor; Development.

\section{Resumen}

Este estudio tuvo como objetivo verificar la relevancia de la expansión de las Indicaciones Geográficas (IG) como una forma de impulsar el desarrollo de la cachaza brasileña. El método elegido para esta investigación es de carácter cualitativo, mediante el uso de técnicas de revisión bibliográfica y documental. La IG de la cachaza en forma de Indicación de Origen para los titulares del sello es de fundamental importancia en la lucha contra las falsificaciones y la identificación para los consumidores como el verdadero origen de las cachazas. las ventajas de registrar la puesta en valor del saber hacer y las técnicas tradicionales, agregando valor, notoriedad, desarrollo territorial y preservación de la cultura, además de demostrar que el producto tiene cualidades. Así, se concluye que la expansión de los registros de IG de cachaza, impulsará el desarrollo de la cachaza y en consecuencia el desarrollo económico del país con la 
reducción de la informalidad en la producción y comercialización de la cachaza, que es un producto de gran relevancia en las áreas blandas. Poder brasileño.

Palabras clave: Indicación geográfica; Cachaza; Desarrollo.

\section{Introdução}

Em 1994, a Organização Mundial do Comércio (OMC) criou o Trips-Trade Related Aspects of Intellectual Property Rights (Acordo sobre Aspectos do Direito de Propriedade Intelectual Relacionados ao Comércio), determinando que os países membros da OMC adotem padrões rígidos de proteção de patentes, tendo como vetor de promoção local as Indicações Geográficas, as quais são conceituadas como o reconhecimento de produtos ou serviços que são originários de um local, o que lhes atribui reputação, valor intrínseco e características atribuídas à origem geográfica que o distinguem em relação aos seus similares disponíveis no mercado (OMPI, 2012).

De acordo com Gurgel (2005), as IGs no Brasil se caracterizam como uma forma legal de proteção concedida a produtos ou serviços que estejam associados a uma determinada região. Neste cenário, a cachaça brasileira, bebida genuinamente nacional, obtida pela destilação do mosto fermentado da cana-de-açúcar, cuja origem remonta aos engenhos de açúcar do período colonial, obteve em 2001 o reconhecimento do Governo Federal, por meio do Decreto 4.062, que estabeleceu o direito de propriedade intelectual a Indicação Geográfica Cachaça do Brasil. Dentre as providências deste decreto, ficou estabelecido que apenas produtores localizados no território brasileiro podem utilizar as expressões "Cachaça", "Brasil" e "Cachaça do Brasil" em suas bebidas.

Em 2007, o INPI reconheceu a primeira Indicação Geográfica de microrregião produtora de Cachaça na modalidade Indicação de Procedência (IP) em Paraty-RJ, em seguida outras microrregiões produtoras buscaram esse registro, no ano de 2012 a Região de Salinas-MG adquiriu o reconhecimento de IP para a cachaça produzida na região e o terceiro registro de IG para cachaça foi concedida para a microrregião de Abaíra-BA (INPI, 2021).

Segundo dados apresentados pelo Anuário da Cachaça de 2021, elaborado pelo Ministério da Agricultura, Pecuária e Abastecimento (MAPA), o Brasil possui 1.131 produtores de Aguardente e Cachaça registrados em 2020, tendo como principais estados produtores: Minas Gerais, São Paulo, Pernambuco, Ceará e Paraíba (IBRAC, 2021). Os estados do Amapá e Roraima não apresentam produtores de cachaça e aguardente registrados (Anuário da cachaça, 2021). Em vista disso, a obtenção da expansão do reconhecimento das Indicações Geográficas para os principais produtores de cachaça no Brasil pode vir possibilitar o desenvolvimento econômico e vantagem competitiva do produto.

Para Glass e Castro (2009), as IGs se constituem como uma promoção mercadológica coletiva, ressaltando lugares, produtos e cultura, com o intuito de obterem o reconhecimento no mercado com a divulgação de produtos que apresentem garantia de qualidade e procedência. A regulamentação brasileira e, por consequência, a utilização de indicações geográficas não são novidades, mas vem evoluindo atualmente (Valente et al, 2020). Os autores corroboram com a afirmação do então Presidente do Sebrae Carlos Melles sobre a expansão das Indicações Geográficas como estímulos ao desenvolvimento local, a partir da valorização dos produtos, preservação cultural, alavancagem do turismo, geração de emprego e renda, ao proporcionar mudanças significativas nas comunidades em todo o Brasil (Agência Sebrae de Notícia, 2021). Logo pode-se afirmar que esse estudo se justifica, pois há poucas pesquisas que analisam os avanços no reconhecimento de novas Indicações Geográficas da cachaça para refletir no desenvolvimento regional, e consequentemente, para o Brasil.

Desse modo levantou-se o seguinte problema de pesquisa: Quais as provocações das Indicações Geográficas para os dominantes produtores de cachaça em termos de vantagens competitivas, a fim de disseminar a progressão loco regional e econômico destes locais?

Neste sentindo, tem-se como justificativa da pesquisa a contribuição para a sociedade com a disseminação do tema, 
juntamente com a valorização da cachaça, produto exclusivo da cultura brasileira, para a academia como referencial para futuros estudos específicos na área de IG de cachaça a qual possui um déficit de pesquisas e para o desenvolvimento locoregional demonstrando que a expansão das concessões dos registros de IG proporcionam vantagens comprovadas com as IG de cachaça existentes que beneficiam todos os atores envolvidos com as IG.

Para se ter uma concepção financeira, conforme pode ser observado através de dados da Organização das Nações Unidas para Alimentação e Agricultura (FAOSTAT), publicados no portal internacional Mongabay, em 2012 o ranking dos produtores de cana-de-açúcar do mundo possuía como cinco primeiros colocados: Brasil, Índia, China, Tailândia e México. Nesse cenário, os estados brasileiros que mais se destacam na produção de cachaça são: Ceará, Paraíba, Pernambuco, Minas Gerais e São Paulo, porém apenas Minas Gerais possui IG (Sebrae, 2016). Nesta perspectiva, e quando se compara a renda per capita de regiões com IGs de cachaça que são Rio de Janeiro ( $\mathrm{R} \$ 1723,00)$; Minas Gerais ( $\mathrm{R} \$ 1314,00)$ e Bahia (R $\$ 965,00)$, com as regiões que não tem IG: Paraíba ( $\mathrm{R} \$ 862,00)$, Pernambuco $(\mathrm{R} \$ 892,00)$, Ceará $(\mathrm{R} \$ 1.028,00)$ e São Paulo $(1814,00)$, sugerindo-se que a busca pela IGs destas regiões fortes produtoras de cachaça, garantam a credibilidade do seu produto, potencializando seu valor comercial e possivelmente, impulsionando aspectos relativos a ambiente econômico e de desenvolvimento social apontando a relevância do tema e a necessidade de expor seu estudo (IBGE, 2020).

Sendo assim, o objetivo geral desta pesquisa é evidenciar os melhoramentos alcançados pelos detentores atuais dos registros de IG de cachaça, evidenciando as vantagens competitivas das IGs da cachaça relevantes ao produto, detentores do registro, internacional e assim denotar o potencial do crescimento das Indicações Geográficas para instigar o desenvolvimento da cachaça, com assistência de uma Análise SWOT.

Dessa forma, o presente estudo está estruturado em quatro seções. Na primeira seção contextualiza as Indicações geográficas no Brasil e as vantagens competitivas das IGs da cachaça no Brasil, relacionadas ao produto, detentores do registro, localização e mercado internacional. A seguir, foi realizada a segunda seção a qual é composta da metodologia utilizada na investigação. A terceira seção contextualiza os resultados da pesquisa através das discussões dos argumentos e dados apresentados no contexto das IGs cachaça no Brasil, desenhadas pela Análise SWOT. Por fim, a quarta seção apresentase a conclusão, limitações e recomendações de trabalhos futuros.

\section{Metodologia}

Este trabalho foi desenvolvido em duas etapas. A primeira foi constituída com base nas pesquisas bibliográficas e documentais relacionadas ao referencial teórico do estudo, assim, pode-se apresentar os benefícios obtidos pelos detentores atuais dos registros de IG de cachaça, relatando as vantagens competitivas das IGs da cachaça pertinentes ao produto, detentores do registro, localização e mercado internacional e assim comprovar o potencial da expansão das Indicações Geográficas para impulsionar o desenvolvimento da cachaça, objeto deste estudo e com isso realizar a segunda etapa da pesquisa com a construção da Análise SWOT [Strengths (Forças), Weaknesses (Fraquezas), Opportunities (Oportunidades) e Threats (Ameaças)], onde retratou-se as implicações das Indicações Geográficas para os principais produtores de cachaça em termos de vantagens competitivas, a fim de expandir o desenvolvimento loco regional e econômico destes lugares.

Segundo Gil (2009), a pesquisa bibliográfica possui o objetivo de abordar o que já foi feito sobre o tema estudado, e a análise é obtida principalmente em livros, artigos científicos e anais de congressos. No entanto, a pesquisa documental possui como função principal a obtenção de informações que estejam fora do centro acadêmico. Conforme relatado por Fonseca (2002), a pesquisa documental recorre a fontes como tabelas estatísticas, jornais, revistas, relatórios, documentos oficiais e dentre outras bases de dados que tratam do tema em questão. 
De acordo com Malhotra (2011), trata-se de uma abordagem qualitativa para alcançar seus objetivos da pesquisa e responder a problemática do estudo que trata em verificar o quanto a expansão dos registros de Indicações Geográficas da cachaça para os principais estados produtores que ainda não possuem IGs, são importantes para criar vantagens competitivas do produto, aliado ao desenvolvimento loco regional e econômico. No intuito de assegurar a qualidade da presente pesquisa, foram utilizadas as bases de dados (Google Scholar, Web of Science e Scopus) essa escolha permitiu o acesso a um acervo maior de buscas com o tema pretendido.

Na definição de campo de busca, utilizou-se como base as palavras-chaves: I- geographical nndication, II- cachaça, III- development, IV- development regional e V-development economic. Depois de obtidos os resultados das buscas, estes foram analisados quanto à sua relevância, de forma a determinar se deveriam ser considerados como estudos primários ou desconsiderados.

A segunda etapa da pesquisa foi realizada a análise de SWOT, descrevendo-se as implicações das Indicações Geográficas para os principais produtores de cachaça em termos de vantagens competitivas, a fim de expandir o desenvolvimento loco regional e econômico destes lugares, problemática do estudo.

De acordo com Bicho e Baptista (2006), a análise de SWOT é a realização da avaliação competitiva das organizações no mercado através do acompanhamento dos ambientes interno relacionados aos pontos fortes e pontos fracos; e ambientes externo constituído por oportunidades e ameaças. Desse modo, foi realizado uma análise do ambiente interno e externo dos detentores de IGs da cachaça para verificar as oportunidades e ameaças e demonstrar os resultados advindos da expansão das IGs, sendo possível com o desenvolvimento deste estudo realizar a análise da expansão das IGs.

\section{Resultados e Discussão}

A partir dos artigos compilados, foi realizado a análise de SWOT representada no quadro 1, com o intuito de descrever os resultados, considerando a problemática do estudo e assim poder verificar quais as implicações das Indicações Geográficas para os principais produtores de cachaça em termos de vantagens competitivas, a fim de expandir o desenvolvimento loco regional e econômico destes lugares, assim foi confrontado a realidade encontrada com a literatura existente. Finaliza-se este capítulo com a discussão dos resultados, baseados da análise de SWOT realizada.

Quadro 1- Análise de SWOT da expansão das IGs da cachaça.

\begin{tabular}{|c|c|c|}
\hline \multirow{8}{*}{$\begin{array}{l}\text { AMBIENTE } \\
\text { INTERNO }\end{array}$} & FATORES POSITIVOS & FATORES NEGATIVOS \\
\hline & PONTOS FORTES & PONTOS FRACOS \\
\hline & Valorização do produto & Alto grau de informalidade \\
\hline & Turismo rural & Falta de cooperação entre os produtores \\
\hline & Preservar o saber fazer & ---------------- \\
\hline & Gestão e controle coletivo & ------------- \\
\hline & Garantia de proteção ao nome & ---------------- \\
\hline & Aumento das vendas dos produtos & ---------------- \\
\hline \multirow{6}{*}{$\begin{array}{l}\text { AMBIENTE } \\
\text { EXTERNO }\end{array}$} & OPORTUNIDADES & AMEAÇAS \\
\hline & Geração de mais empregos & $\begin{array}{l}\text { Falta de recursos financeiros dos micros e } \\
\text { pequeno empresários }\end{array}$ \\
\hline & Movimentar a economia local & $\begin{array}{l}\text { Imagem do produto pouco difundido no } \\
\text { mercado externo }\end{array}$ \\
\hline & Internacionalização do produto & Alta tributação do produto \\
\hline & Movimentar a economia local & --------------- \\
\hline & $\begin{array}{l}\text { Possibilidade do negócio passar de geração para } \\
\text { geração }\end{array}$ & \\
\hline
\end{tabular}

Fonte: Elaborado pelos autores a partir dos dados das pesquisas bibliográfica e documentais (2021). 
Como demonstrado na análise de SWOT acima, coadunam com a proposta de relevância da pesquisa a qual comprova que as IGs para os principais produtores de cachaça existentes possuem vantagens competitivas, a fim de expandir o desenvolvimento loco regional e econômico. Para tanto, as experimentos efetivas das IGs presentes produzem para disparar a expansão das IGs da cachaça para os produtores que ainda não apresentam os registros.Na sequência serão apresentados os resultados referentes a expansão das IGs da cachaça, descrevendo os fatores positivos e fatores negativos.

Em termos de análise do ambiente interno, foram identificados os resultados como pontos fortes (Strengths): Valorização do produto; Turismo rural; Preservar o saber fazer; Gestão e controle coletivo; Garantia de proteção ao nome; e Aumento das vendas dos produtos, para os pontos fracos (Weaknesses): Alto grau de informalidade e Cooperação entre os produtores. Podemos desta forma identificar os fatores críticos de sucesso ou não das IGs de cachaças existentes, obtendo assim o controle como proposta para difusão de informações que devem ser realizadas através de monitoramento periódico por Universidades, Governo e a Indústria da cachaça para alavancar o setor cada vez mais e não perder oportunidades de crescimento.

\subsection{Ambiente Interno - Pontos Fortes}

No que concerne aos fatores positivos de relevância para a obtenção dos registros de IG da cachaça, tem-se a valorização do produto que permitirá a difusão da cultura da cachaça, bebida genuinamente brasileira com a garantia para o consumidor da aquisição de um produto de qualidade e que o mesmo estará contribuindo com a economia da região e a propagação da internacionalização da cachaça. Neste sentido, têm-se o fator positivo de valorização do produto caracterizado como ponto forte para a obtenção do registro de IG para a cachaça coadunam com o ponto de vista de Mascarenhas; Wilkinson (2014), visto que geram valor e auxiliam no desenvolvimento regional.

No que tange ao preservar o saber- fazer, Niederle (2015), sugere que as IGs além de preservar o saber-fazer a produção de forma tradicional, estabelecem padrões normativos a serem seguidos tanto na produção quanto na comercialização dos produtos, o que engendra exigências de adequações a tecnologia e a produtividade, no intuito de aumentarem a qualificação do produto. Sabendo-se que mesmo os engenhos obtendo maquinários sofisticados, a tradição resiste ao tempo e as peculiaridades do cultivo da cana-de-açúcar ao processo produtivo da cachaça, também são vistos como preservação do saber-fazer proporcionando diferencial entre os produtos similares para os detentores dos registros de IGs para a cachaça e que seguem os padrões normativos, mas mantém suas tradições, podendo ser subentendido como uma ameaça da produção em larga escala.

Outro ponto de profunda importância a gestão e controle coletivo fatores que permitem que os detentores dos registros de IGs de cachaças fortaleçam a relação do grupo, aumentando a competitividade e a cooperação, características que resultam em ganhos para todos os envolvidos, fato este que corrobora com o posicionamento de Mafra (2008), quando afirma que as IGs são uma construção coletiva, originada do conhecimento comunitário e não apenas de um produtor.

Já no que concerne ao turismo as IGs nos países em desenvolvimento tem valorizado a expansão de produtos diferenciados e que possuem atratividades turísticas e culturais. Além disso, o turismo contribui de forma significativa para o desenvolvimento regional, valorização do patrimônio histórico e na melhoria da qualidade de vida permitindo geração de emprego e renda (Seabra \& Mariano, 2004).

Diante do fator positivo garantia da proteção do nome, se caracteriza como benefício assegurado para os detentores do registro de IG permitindo a restrição ao uso da IG para os produtores das regiões, evitando o uso indevido do selo.

Assim, com a obtenção desses pontos fortes acarretaram consequentemente no aumento das vendas da cachaça com registros de IG, demonstrando assim o resultado da qualidade e da preservação das características do produto que 
proporcionam segurança aos consumidores e permitem a preservação cultural. Neste contexto, a IG permite ao consumidor a identificação não apenas da procedência do produto, mas reitera que seja de qualidade (Cotton, 2007).

\subsection{Ambiente Interno - Pontos Fracos}

É importante analisar que mesmo o setor produtivo da cachaça tendo um grande potencial de expansão com a concessão do selo de IG, o setor possui entraves discriminados na pesquisa como fatores internos e externos que causam entrave para a realização da expansão das IGs. Para tanto, entende-se que o elevado número de produtores informais que não são registrados no MAPA, estão relacionados ao fator negativo do ambiente externo que são a alta carga tributária, correspondendo a $80 \%$ do volume da bebida engarrafada (IBRAC, 2020). Desse modo, pode ser analisado como o principal gargalo para desestimular a legalização e, consequentemente a expansão das IG para a cachaça.

Para Carrão (2004), a cooperação deve se sobressair ao individual nas empresas. O que não poderá ser diferente na relação entre os produtores de cachaça, os quais devem primar por comportamentos de cooperação e não o individualismo, beneficiando assim a rede de detentores da IG da cachaça como comunidade em que estão inseridos, fator este negativo que implica na recusa dos produtores de cachaça em irem em busca da concessão do registro de IG.

\subsection{Ambiente Externo - Oportunidades}

Tendo como análise do ambiente externo, foi encontrado entre as IGs de cachaça existentes no Brasil os fatores positivos de geração de emprego, onde Rocha (2016) relatou que a IG possibilitou a geração de empregos e os resultados da pesquisa de Silva, Gallego \& Montibeler (2012) verificaram que o Brasil possui um vasto fator a ser explorado no caso da cachaça, podendo este ser um excelente produto a compor a pauta de exportações, contribuindo para o aumento do número de empregos, relevante no setor das cachaças artesanais, e para a crescente economia brasileira. Esses fatores demonstram a significância das IGs para o setor.

Portanto, em se tratando de pontos fortes da economia, Rocha (2016) citou que há uma melhor distribuição do valor agregado ao passo da cadeia produtiva, permitindo assim o desenvolvimento das atividades em zonas rurais desfavorecidas, distribuindo renda e valorizando as habilidades locais, permitindo dessa forma elevar os preços em função da tipicidade do produto, possibilitando a rastreabilidade do produto desde o seu plantio até sua comercialização, oportunizando a expansão do turismo na área, entre outros. Dessa forma demonstrando que fortalece a economia local, visto que intensifica a diversificação e ampliação de mercados.

Segundo Silva, Gallego \& Montibeler (2012) em sua pesquisa sobre a internacionalização da cachaça, relatou que ocorreu um vasto investimento em relação ao controle de qualidade e na elaboração e inovação de marketing para mudar de status e prospectar o mercado internacional. As empresas desenvolveram garrafas e rótulos mais sofisticados, detalhes aprimorados que surtiram vantajosos efeitos na estratégia de internacionalização do produto. Um dos resultados deste estudo Silva, Gallego \& Montibeler (2012) foi que o Brasil possui um grande potencial a ser explorado no setor da cachaça, podendo este ser um importante produto componente da pauta de exportações. Portanto, a importância da internacionalização deste produto e suas vantagens para as organizações.

Em sua pesquisa, Rocha (2016) relata que alguns dos produtores de cachaça já chegaram até sua quinta geração e afirma que em sua maioria, os engenhos já estão na família há gerações, sendo uma cultura passada de pai para filho. Silva, Gallego \& Montibeler (2012, p. 2) confirma que o "conhecimento mais aprofundado abordando assuntos inerentes a uma tradição que foi passada de geração para geração ao longo dos anos". Dessa forma o destaque para a cachaça como forma de estratégia o conhecimento designado de geração para geração. 


\subsection{Ambiente Externo - Ameaças}

Tendo como análise do ambiente externo, foi encontrado entre as IGs de cachaça existentes no Brasil alguns fatores negativos. Valente et. al., (2012) relataram que a indicação geográfica ainda está engatinhando no Brasil para que seja consolidada popularizada, amadurecida e desenvolvido o modelo de indicações geográficas é positivo para que haja maiores investimentos em recursos de pessoas, técnicos e financeiros por parte das empresas competentes, políticas de difusão do tema junto aos consumidores, engajamento com universidades e organizações de pesquisa e fomentação do setor agropecuário. Esses fatos são importantes para alcançar a indicação geográfica e transformar em uma ferramenta de desenvolvimento tanto econômico quanto social.

Mascarenhas \& Wilkinson (2014) retratam que ao se obter o selo de indicação geográfica é um passo relevante nesse processo de superestima do território, por sua vez, ao resgatar o modo de tradicional de produção, traços culturais, dessa forma agregar valor e auxiliar no desenvolvimento rural e de estratégia de posição de mercado doméstico e internacional. O IG causa a difusão no mercado trazendo com isso uma melhor imagem do produto.

Em sua pesquisa Rocha (2016) revelou sobre os trâmites no Congresso Nacional, da volta da cachaça ao Simples, esse imposto simplifica as cobranças tributárias de micro e pequenas empresas, que totalizam hoje $99 \%$ da produção da bebida, já que $81 \%$ do valor da cachaça se equipou a tributos. Essa via visa, além de diminuir a carga de tributos, retirar grande e maior parte das organizações do lado informal, que chega a 85\% dos produtores no Brasil (Cenário MT, 2015). Nesse contexto muitos produtores desistem da produção por causa da alta tributação deste produto, além da concorrência desleal, falta de capital de giro e perda de competividade.

\section{Conclusão}

Conclui-se que a propriedade intelectual (PI) tem sido um primordial meio para o desenvolvimento socioeconômico mundial. Sendo assim, as IGs para a cachaça bebida genuinamente brasileira de tradição histórica e relevância econômica para o país possui muitas oportunidades de expansão ainda não exploradas pelo setor produtivo.

As pesquisas realizadas apontam que os três registros de IG da cachaça no Brasil possuem experiências positivas que proporcionam benefícios não somente aos produtores, cadeia produtiva, mas para todo o território de abrangência da concessão, gerando a satisfação dos produtores detentores dos registros em serem associados, vantagens competitivas, aumento na comercialização dos produtos, aumento de investidores nacionais e internacionais, fortalecimento da comercialização da cachaça com ações de marketing em que os custos são divididos entre os produtores associados, cooperação entre os produtores, conquista de novos mercados internos e externos, valorização e diferenciação do produto com registro de IG dos similares. Para as localidades onde as IGs estão inseridas são beneficiadas com o crescimento do turismo, geração de emprego e renda, modificação nos contextos econômicos, sociais e culturais da região e contribui para o consumidor com a qualidade do produto registrado com selo de indicação de procedência e qualidade da cachaça percebida pelo consumidor.

Nesta conjuntura, a expansão dos registros de IGs da cachaça para os principais estados produtores que ainda não possem IG relacionando com as experiências positivas dos registros atuais permitirá impulsionar o desenvolvimento da cachaça e consequentemente o desenvolvimento econômico para o país com a diminuição da informalidade na produção e comercialização da cachaça que é um produto de grande relevância no soft power brasileiro, visto que tratam-se de regiões vulneráveis e que poderiam se valer do IGs como forma de impulsionar o ambiente local. A limitação desta pesquisa foi a ausência de trabalhos atuais específicos sobre o tema IGs de cachaça. Sugere-se para pesquisas futuras que seja realizado estudo de caso das IG de cachaça com produtores que não possuem IGs. 


\section{Referências}

A cachaça no Brasil. (2020) Dados de Registro de cachaças e aguardentes. Junho.

Agência Sebrae de Notícias. (2021) Oitenta novas indicações geográficas no país são identificadas com potencial de serem reconhecidas. Junho.

Aprovale. (2021) Indicações geográficas. Junho.

Barahona, I., Sanmiguel Jaimes, E. M., \& Yang, J. (2020). Sensory attributes of coffee beverages and their relation to price and package information: A case study of Colombian customers' preferences. Food Science \& Nutrition, 8(2), 1173-1186.

Bicho, L.; Baptista, S. (2006). Modelo de Porter e Análise de SWOT: Estratégias de Negócios. Instituto Politécnico de Coimbra.

Boncinelli, F., Dominici, A., Gerini, F., \& Marone, E. (2019). Consumers wine preferences according to purchase occasion: Personal consumption and giftgiving. Food Quality and Preference, 71, 270-278.

Bowen, S. (2010). Embedding local places in global spaces: Geographical indications as a territorial development strategy. Rural Sociology.

Brasil. (2001). Decreto n. 4.062, de 21 de dezembro de 2001. Define as expressões "cachaça", "Brasil” e "cachaça do Brasil" como indicações geográficas e dá outras providências.

Brasil. (1996). Lei n.9279, de 14 de maio de 1996. Regula direitos e obrigações relativos à propriedade industrial.

Brasil. (2001). Decreto n. 4.062, de 21 de dezembro de 2001. Define as expressões "cachaça", "Brasil” e "cachaça do Brasil”" como indicações geográficas e dá outras providências.

Barbosa, D. B. (2003). Uma Introdução à Propriedade Intelectual. 2. Ed. Rio de Janeiro: Editora Lumen Juris.

Carrão, A. M. R. (2004) Cooperação entre empresas de pequeno porte. Revista de Administração da USP, 39(2), $186-185$.

Castro, V. A. \& Giraldi, J. M. E. (2018). Estratégias de marcas para setores brasileiros: diferenças conceituais entre indicação geográfica, marca coletiva e setorial. Revista Espacios. 39(33), 1-14.

Caldas, A. S. (2013) Indicações Geográficas: marco regulatório e distribuição espacial. In: Caldas, A. S; Brito, C.; Fonseca, A. A. M.; Pertile, Noeli. (Org.). Gestão do Território e Desenvolvimento: novos olhares e tendências. Salvador: JM, 1(1), 127-152.

Caldas, A. D. S., de Araújo, C. C., \& Coury, R. D. L. M. (2018). As Indicações Geográficas (IGS) como estratégia de desenvolvimento territorial: desafios e potencialidades no distrito de Maragogipinho, Aratuípe, BA. RDE-Revista de Desenvolvimento Econômico, 3(38).

Calegario, C. L. L. (2006). Comercialização e exportação da cachaça. In: Cardoso, M. G. Produção de aguardente de cana. 2. ed. Lavras: Editora UFLA. Cavalcante, E. D. (2013). A cachaça e o desenvolvimento turístico: uma análise das representações do espaço e dos atores envolvidos na atividade turística no brejo paraibano. Dissertação (mestrado). Universidade Estadual da Paraíba.

CenárioMT. (2015). Produtores de Cachaça defendem volta do "'"Simples"'". http://www.cenariomt.com.br/noticia/489290/produtores-de-cachacadefendemvolta-do-simples.html>.

Centro Brasileiro de Referência da Cachaça (2015). Campanha para a Valorização da Cachaça. Junho

Cerdan, C. (2013). Desafios institucionais e organizacionais ao desenvolvimento das IGs no Brasil. In: NIEDERLE, P.A. (Org.). Indicações geográficas e estratégias de desenvolvimento territorial. Porto Alegre: Editora da UFRGS.

Cotton, A. P. (2007) 123 Years at the Negotiating Table and Still No Dessert - The Case in Support of TRIPS Geographical Indication Protections. ChicagoKent Law Review. 8(3), 1295-1316.

Conceição, V. S. et al. (2020). A Indicação Geográfica da Cachaça: Um instrumento de desenvolvimento regional e de Inovação. Braz. J. of Develop., (6)6, $35137-35155$

Data Sebrae (2018). IG-Paraty. Junho.

Dortzbach, D., Machado, L. N., Loss, A., \& Vieira, E. (2020). Influência do meio geográfico nas características do mel de melato da bracatinga. Research, Society and Development, 9(9), e198997191. https://doi.org/10.33448/rsd-v9i9.7191

Dos Anjos, F. S., da Silva, F. N., \& Caldas, N. V. (2020). Indicações geográficas, capital social e desenvolvimento territorial. Redes (St. Cruz Sul, Online), 25(2), 721-743.

Fonseca, J. J. S. (2002). Metodologia da pesquisa científica. Fortaleza: UEC.

Gatto, D. B., Clauzet, M., \& Lustosa, M. C. (2019). Governança ambiental e Indicação Geográfica: o caso da denominação de origem manguezais das alagoas. Desenvolvimento Regional em Debate, 9(2), 229-247.

Giesbrecht, H. O; Minas, R.B.A; Gonçalves, M. F.W; Schwanke, F.H. (2014). Indicações geográficas brasileiras. Brasília: Sebrae INPI.

Giesbrecht, H. O. et al. (2016). Indicações geográficas brasileiras. 5. ed. Brasília: Serviço Brasileiro de Apoio às Micro e Pequenas Empresas - SEBRAE; Instituto Nacional da Propriedade Industrial (INPI), 2000(327).

Gil, A. C. (2009). Como elaborar projetos de pesquisa. São Paulo: Editora Atlas. 
Glass, R. F. e Castro, A. M. G. (2009). As indicações geográficas como estratégia mercadológica para vinhos. Embrapa Informação Tecnológica Brasília, DF.

Gonçalves, L. A. S.; Almeida, B. A. Bastos, E. M. S. (2018). Panorama das Indicações Geográficas no Brasil. Revista de Desenvolvimento Econômico - RDE, 3(41), 130-144.

Gurgel, V. A. (2005). Aspectos Jurídicos da Indicação Geográfica In. SEBRAE Valorização de produtos com diferencial de qualidade e identidade: indicações geográficas e certificações para competitividade nos negócios. Organizado por Vinícius Lages, Léa Lagares e Chistiano Lima Braga. Brasília.

Harbison, J. R. \& Pekar Junior, P. (1999). Alianças estratégicas: quando a parceria é a alma do negócio e o caminho para o sucesso. São Paulo: Futura.

Hughes, J; Beresford, L; Kur, A; Plevan, K; Scafidi, S. (2007). That's a Fine Chablis You're not drinking: The Proper Place for Geographical Indications in Trademark Law. Fordham Intellectual Property, Media and Entertainment Law Journal, 17(4), 933-978.

IBGE-Instituto Brasileiro de Geografia e Estatística. (2020). PIB 2020. Junho.

IBGE-Instituto Brasileiro de Geografia e Estatística. (2020). Renda Per Capita 2020. Junho.

IBRAC- Instituto Brasileiro da Cachaça. (2021). Mercado Interno. Junho.

IBRAC- Instituto Brasileiro da Cachaça (2021). O IBRAC. Junho.

INPI- Instituto Nacional da Propriedade Industrial. (2018). Instrução Normativa $n^{o}$ 95/ 2018.

INPI - Instituto Nacional da Propriedade Intelectual. (2015). Guia Básico - Indicação Geográfica. Julho.

Josling, T. (2006). The war on terroir: geographical indications as a transatlantic trade conflict. Journal of Agricultural Economics, Malden,

Krajnc, B., Bontempo, L., Luis Araus, J., Giovanetti, M., Alegria, C., Lauteri, M., ... Ogrinc, N. (2020). Selective Methods to Investigate Authenticity and Geographical Origin of Mediterranean Food Products. Food Reviews International, 1-27.

Kotler, P \& Keller, L. K. (2006). Administração de marketing. 12. Ed. São Paulo: Pearson Prentice Hall.

Mafra, L.A.S. (2008). Indicação Geográfica e construção do mercado: a valorização da origem no cerrado mineiro. Tese (doutorado). Universidade Federal Rural do Rio de Janeiro, Instituto de Ciências Humanas e Sociais.

MAPA- Ministério da Agricultura, Pecuária e Abastecimento. (2017) O que é indicação geográfica? Como obter o registro? Junho.

Mapa da Cachaça (2020). A indicação geográfica da cachaça de Paraty. Julho.

Malhotra, N, K. (2011). Pesquisa de Marketing: foco na decisão. 3 ed. São Paulo: Pearson Prentice Hall.

Mascarenhas, G.C.C.; Wilkinson, J. (2014). Indicações geográficas em países em desenvolvimento: potencialidades e desafios. Revista de Política Agrícola, ano XXIII, n.2.

Martins, I. S. S. (2019) Iniciativas para a Proteção das Indicações Geográficas no Brasil. Junho.

Mendonça, D., Procópio, D. P., \& Santos Corrêa, S. R. (2019). A contribuição das indicações geográficas para o desenvolvimento rural brasileiro. Research, Society and Development, 8(7), e41871152.

Mercosul. (1995). Protocolo de harmonização de normas sobre a Propriedade Intelectual no Mercosul, em matéria de marcas, indicações de procedência e denominações de origem.

Nascimento, J. S.; Fialho, A. S.; Nunes, G. S., \& Bandeira, M. G. A. (2012). Indicações geográficas: agregação de valor aos produtos brasileiros e maranhenses. Revista GEINTEC: Gestão, Inovação e Tecnologias, 2 (4), 353-364.

Niederle, P. A (2010). Compromissos para a qualidade: projetos de indicação geográfica para vinhos no Brasil e na França. Tese (doutorado). Universidade Federal Rural do Rio de Janeiro.

OMPI- Organização Mundial de Propriedade Intelectual (OMPI). 2021. Indicações Geográficas. Junho.

Pellin, V. (2017). Indicações Geográficas e Desenvolvimento Regional no Brasil: a atuação dos principais atores e suas metodologias de trabalho. Research, Society and Development, 33(21), 33-38.

Pellin, V. (2019). Indicações Geográficas e desenvolvimento regional no Brasil: a atuação dos principais atores e suas metodologias de trabalho. Interações (Campo Grande), 20, 63-78.

Rocha, E. D. L. (2016). Propriedade intelectual por indicação geográfica: O caso da cachaça do Brejo paraibano. 132 f. Dissertação (Mestrado em Administração) - Universidade Federal da Paraíba, João Pessoa.

Seabra, G.; Mariano, G. (2004). Turismo rural no agreste Pernambucano: o caminho das pedras é também das flores e dos frutos. In:Congresso Internacional sobre TurismoRural e Desenvolvimento Sustentável, IV, Joinville. 2004 Anais. Joinville: Instituto Superior e Centro Educacional Luterano Bom Jesus/Ielusc.

Sebrae. (2016). Cachaça: Indicações Geográficas Brasileiras. Junho 
Research, Society and Development, v. 10, n. 12, e323101220682, 2021

(CC BY 4.0) | ISSN 2525-3409 | DOI: http://dx.doi.org/10.33448/rsd-v10i12.20682

Silva, T. C. P., Gallego, E. S., \& Montibeler, E. E. (2012). Exportação da cachaça: uma análise do método SWOT para a internacionalização empresarial. Observatorio de la Economía Latinoamericana, (176).

Valente, M. E. R., de Andrade Neves, N., Perez, R., de Moraes Valente, L. R. R., de Lima, J. E., \& Chaves, J. B. P. (2020). Indicação geográfica e qualidade de cachaças segundo a percepção de apreciadores da bebida. Research, Society and Development, 9(10), e2989108365-e2989108365.

Valente, M. E. R.; Perez, R.; Ramos, A. M.; Chaves, J. B. P. (2012). Indicação geográfica de alimentos e bebidas no Brasil e na União Europeia. Ciência Rural, v. 42, n. 3, p. 551-558.

Van Caenegem, W., Cleary, J.A, \& Drahos, P. (2014). Pride and profit: Geographical indications as regional development tool in Australia. Journal of Economica \& Social Policy. 16(1), 90-114. 